\title{
Survivin promoter-based conditionally replicative adenoviruses target cholangiocarcinoma
}

\author{
ZENG BIAN ZHU ${ }^{1}$, YABING CHEN ${ }^{2}$, SHARMILA K. MAKHIJA ${ }^{3}$, BAOGEN LU ${ }^{1}$, \\ MINGHUI WANG ${ }^{1}$, ANGEL A. RIVERA ${ }^{1}$, MASATO YAMAMOTO ${ }^{1}$, \\ SHUYI WANG ${ }^{4}$, GENE P. SIEGAL ${ }^{5}$, DAVID T. CURIEL ${ }^{1}$ and JAY M. McDONALD ${ }^{2,6}$
}

\begin{abstract}
${ }^{1}$ Division of Human Gene Therapy, Departments of Medicine, Obstetrics and Gynecology, Pathology, and Surgery, Gene Therapy Center, University of Alabama at Birmingham; ${ }^{2}$ Department of Pathology, University of Alabama at Birmingham; ${ }^{3}$ Division of Gynecologic Oncology, ${ }^{4}$ Howard Hughes Medical Institute and Departments of Medicine, ${ }^{5}$ Departments of Pathology, Cell Biology, and Surgery, Gene Therapy Center, University of Alabama at Birmingham; ${ }^{6}$ Veterans Administration Medical Center, Birmingham, AL, USA
\end{abstract}

Received March 30, 2006; Accepted June 8, 2006

\begin{abstract}
Cholangiocarcinoma is a highly malignant neoplasm with no effective treatment. Conditionally replicative adenoviruses (CRAds) represent a promising new modality for the treatment of cancer in general. A key contribution in this regard was the introduction of tumor-selective viral replication for amplification of the initial inoculum in the neoplastic cell population. Under ideal conditions following cellular infection, the viruses replicate selectively in the infected tumor cells and kill the cells by cytolysis, leaving normal cells unaffected. However, to date there have been two limitations to the clinical application of these CRAd agents, i.e. poor viral infectivity and tumor specificity. Here we report the construction of three new CRAd agents, CRAd-S.RGD, CRAd-S.F5/3 and CRAd-S.pk7, in which the tumor specificity is regulated by a tumor-specific promoter, the survivin promoter, and the viral infectivity is enhanced by incorporating a capsid modification (RGD, F5/3 or pk7) in the adenovirus fiber region. These CRAd agents effectively target cholangiocarcinoma cells, induce strong cytoxicity in these cells in vitro and inhibit tumor growth in a murine xenograft model in vivo. In addition, the survivin promoter has extremely low activity both in the non-transformed cell line, HMEC, and in human liver tissue. Our results suggest
\end{abstract}

Correspondence to: Dr Zeng Bian Zhu, Division of Human Gene Therapy, Gene Therapy Center, BMR II 534, 2172, 901 19th Street S., University of Alabama at Birmingham, Birmingham, AL 35291, USA

Abbreviations: bp, base pairs; CMV, cytomegalovirus; CRAd, conditionally replicative virus; MOI, multiplicity of infection; pfu, plaque-forming units; vp, viral particles, TSP, tumor-specific promoter

Key words: survivin gene, tumor-specific promoter, cholangiocarcinoma, adenoviral vector, conditionally replicative adenovirus that the survivin-based CRAds are promising agents for targeting cholangiocarcinoma with low host toxicity. Such results should provide important insights into the identification of novel therapeutic strategies for cholangiocarcinoma.

\section{Introduction}

Cholangiocarcinoma is a malignancy derived from the human biliary tract epithelium. It is a highly lethal disease with a poor long-term prognosis and few effective treatments (1-4). Cholangiocarcinoma represents $20 \%$ of all hepatobiliary malignancies in the United States, a number which is increasing every year (5). In these patients, only $30 \%$ are candidates for attempted curative surgical resection at present and another $70 \%$ are found to have occult metastatic or advanced local disease, precluding curative resection. The average 5-year survival is only $10 \%$, with a median survival of 1.5 years. Even after resection, the five-year survival rate is only $20-40 \%$, and chemotherapy and radiotherapy have limited efficacy (6). Clearly, novel treatment modalities which increase efficacy are needed.

Adenovirus (Ad)-mediated gene therapy has been demonstrated to be a powerful tool to target tumor cells and can infect a broad range of human cells, both dividing and nondividing, with high gene transfer efficiency $(7,8)$. In addition, wild-type adenoviruses have a low pathogenicity, typically causing only mild 'cold-type' symptoms in the human host. The use of conditionally replicative adenovirus (CRAd) represents a novel approach to treat neoplastic diseases, including malignant cholangiocarcinoma.(9)

Conditionally replicative adenoviruses target tumor cells leading to their death via a direct consequence of viral replication (10). It is apparent that the specificity of the viral agent for achieving tumor cell killing via replication ('oncolysis') is the functional key to successful exploitation of these agents for therapy. To this end, an ideal viral agent would, thus, possess two characteristics: i) high infectivity with which viral vectors would have the capacity to infect tumor versus non-tumor cells; and ii) tumor specificity with 
which viral vectors would possess a relative preference for replication in tumor versus non-tumor cells. However, both viral infectivity and specificity have been relatively poor when using current applicable CRAd vectors. To develop enhanced infectivity and tumor-specific CRAd agents for cholangiocarcinoma, better constructs are needed.

In order to overcome these two disadvantages, poor infectivity and specificity, many approaches have been described. For example, cells may be resistant to Ad infection due to their lack of the coxsackie adenovirus receptor (CAR) on the surface of tumor cells resulting in poor infectivity (11). To circumvent this, genetic and immunologic alterations to the virus fiber which utilize CAR-independent pathways have been identified. An example of this is the utilization of the RGD motif in the fiber knob of the Ad. This capsid modification appears to facilitate Ad binding and entry into tumor cells via integrin receptors that are abundantly expressed on tumor cells (12). Additional capsid modifications have been explored to obtain infectivity enhancement of Ads including AdF5/3 (13), Ad5-pk7 (14) and Ad5-CK (15). Transcriptional targeting exploits promoters that display preferentially in tumor cells but not in normal host cells. This has been described as an efficient strategy to increase viral replication specificity $(16,17)$. An ideal tumor-specific promoter (TSP) for transcriptional targeting exhibits selective high activity in tumor cells (termed a 'tumor on' phenotype). To mitigate hepatoxicity upon systemic delivery, candidate promoters additionally need to exhibit low activity in liver (termed a 'liver off' phonotype). To develop TSP-CRAds, one of the most widely used methods is to drive Ad E1 gene expression with a selected promoter, as Ad E1 is the main element which drives viral replication. In this method, a CRAd replicates only in tumor cells, killing cells by oncolysis, but not in normal host cells, thereby avoiding the toxicity of the CRAd agent. Many TSPs have been explored for specific cancers, such as prostatespecific antigen (PSA) for prostate cancer, and $\alpha$-fetoprotein (AFP) promoter for hepatocarcinoma $(18,19)$. Recently we reported a novel TSP, the survivin promoter, which showed a tumor on/liver off phenotype in vitro and in vivo (17). This promoter has also been reported to exhibit radiation response elements and cisplatin sensitivity capabilities $(20,21)$. Therefore, the survivin promoter is an excellent candidate to drive E1 expression in development of a new CRAd agent. Kamizono et al (22) and our group (23) have described the survivin-CRAd in which the Ad E1 is driven by the survivin promoter as a multiple tumor-specific promoter target.

We have previously subcloned and characterized the cholangiocarcinoma cell line, SK-ChA-1. Tumorigenicity studies have demonstrated that a subclone of SK-ChA-1 can grow as tumors in nude mice (24). Therefore, as a proof-ofprinciple we developed Ad agents to target these tumors.

We further constructed CRAd vectors in which the Ad E1 gene was regulated by using the survivin promoter as a TSP, viral infectivity was enhanced with a capsid modification (RGD, pk7 or F5/3), and the Ad vector targeting to cholangiocarcinoma cells occured via a CAR-independent pathway. We verified that these infectivity-enhanced and tumorselected vectors, specifically CRAd-S.F5/3, replicate in cholangiocarcinoma cells, kill tumor cells and inhibit tumor growth in a xenograft animal model. We also showed that the survivin promoter had extremely low activity compared to the CMV promoter in an in vitro human liver slice hepatotoxicity model although they have similar oncolytic activity in cholangiocarcinoma cells. The data indicated that CRAd-S.F5/3 is an excellent candidate for translation into a clinical trial for treatment of cholangiocarcinoma in patients.

\section{Materials and methods}

Cells, tissues and animals. Human cholangiocarcinoma cells derived from SK-ChA-1 were characterized and described previously (25). Cells were grown in RPMI-1640 complete supplemented with $10 \%$ fetal calf serum, penicillin (100 IU/ $\mathrm{ml})$, and streptomycin $(100 \mu \mathrm{g} / \mathrm{ml})$ at $37^{\circ} \mathrm{C}$ in a $5 \% \mathrm{CO}_{2}$ environment under humidified conditions. The normal cell line, human mammary epithelial cells (HMEC), as control cells, was purchased from the Cambrex BioScience Company (Walkersville, MD) and cultured in medium purchased from the same company.

Human liver samples were obtained from hepatectomy remnants (donor) not needed for diagnostic purpose after liver transplantation following IRB approval. To generate liver tissue slices, tissue was cut in consecutive 0.5 -mm thick slices using the Krumdieck tissue slicer (Alabama Research Development, Munford, AL). Sequential slices were then cultured in 24-well plates in RPMI medium supplemented with $10 \%$ bovine fetal serum, $100 \mathrm{U} / \mathrm{ml}$ penicillin, $100 \mu \mathrm{g} / \mathrm{ml}$ streptomycin and $5 \mu \mathrm{g} / \mathrm{ml}$ insulin. Cultures were maintained at $37^{\circ} \mathrm{C}$ in a humidified atmosphere of $95 \%$ air and $5 \% \mathrm{CO}_{2}$. Three tissue slices were examined per group.

Female BALB/c nude mice (6-8 weeks of age, Charles River, Wilmington, MA) were used in the in vivo experiments. All animals received humane care based on the guidelines set by the American Veterinary Association. All experimental protocols involving live animals were reviewed and approved by the Institutional Animal Care and Use Committee of the University of Alabama at Birmingham.

Recombinant adenoviruses. Ad5-CMV, Ad5-Cox-2, Ad5CXCR4, Ad5-EGP-2, Ad5-HPR, Ad5-SLPI, Ad5-MsLn, and Ad5-Survivin (Ad5-S) are replicative-defective Ads containing a luciferase reporter gene driven by the tumor-specific promoters, Cox-2 (26), CXCR4 (16), HPR (27), SLPI (28), MsLn (29) and survivin (23) and a control CMV promoter, respectively, in the E1 region and have been described previously. These Ads were used in this study for evaluating the transcriptional activity of the TSPs by means of the expression of the luciferase reporter gene in cholangiocarcinoma cells.

Ad5.RGD, Ad5pk7, Ad5F5/3, and Ad5pk7.RGD are replicative-defective Ads containing a luciferase reporter gene driven by the CMV promoter in the E1 region and incorporating a capsid modification, RGD (30), pk7 (14), F5/3 (31), or pk7.RGD (14) respectively as described previously. These Ads were used in this study for evaluating the transductional activity of the capsid modifications by means of the expression of a luciferase reporter gene in cholangiocarcinoma cells.

All the Ads are listed in Table I. The viruses are all isogenic and were propagated in 293 cells and purified by double $\mathrm{CsCl}$ density centrifugation. Of note, the firefly luciferase gene 
Table I. The characteristics of adenoviral vectors used in this study.

\begin{tabular}{lllllcc}
\hline Virus name & Promoter & Reporter & E1 & E3 & Modification & Replication competent \\
\hline Ad5-CMV & CMV & Luciferase & No & No & No & No \\
Ad5-Cox-2 & Cox-2 & Luciferase & No & No & No & No \\
Ad5-CXCR4 & CXCR4 & Luciferase & No & No & No & No \\
Ad5-EGP-2 & EGP-2 & Luciferase & No & No & No & No \\
Ad5-HPR & HPR & Luciferase & No & No & No & No \\
Ad5-SLPI & SLPI & Luciferase & No & No & No & No \\
Ad5-Mk & Midkine & Luciferase & No & No & No & No \\
Ad5-Survivin & Survivin & Luciferase & No & No & No & No \\
Ad5.RGD & CMV & Luciferase & No & No & RGD4C & No \\
Ad5.F5/3 & CMV & Luciferase & No & No & F5/3 & No \\
Ad5.pk7 & CMV & Luciferase & No & No & pk7 & No \\
Adwt & Native & No & Yes & Yes & No & Yes \\
CRAd-S.RGD & Survivin & No & Yes & Yes & RGD4C & Yes \\
CRAd-S.FS/3 & Survivin & No & Yes & Yes & F5/3 & Yes \\
CRAd-S.pk7 & Survivin & No & Yes & Yes & pk7 & Yes \\
\hline
\end{tabular}

incorporated into the Ads contained a modified coding region for firefly luciferase (pGL3; Promega) that had been optimized for monitoring transcriptional activity in transfected eukaryotic cells. The luciferase activity of cells infected with one of the Ads was normalized to fold-activity of cells infected with Ad5-CMV.

Physical particle concentration (v.p./ml) was determined by O.D. $260 \mathrm{~nm}$ reading. All experiments were based on v.p. numbers, although a plaque assay was performed to ensure sufficient quality of the virus preparation. The ratios of v.p./pfu (plaque forming units) were 19-40 among all Ad vectors.

Quantitative real-time PCR (QRT-PCR) for detection of the expression of the survivin, Ad5 E1 and E4 genes. For survivin gene expression, total cellular RNA was extracted from $5 \times 10^{5}$ cholangiocarcinoma cells or HMEC cells using the RNeasy mini kit (Qiagen, Valencia, CA), followed by treatment with DNase to remove any possible contaminating DNA from the RNA samples. The fluorescent TaqMan probe (6FAMTGACGACCCCATAGAGGAACATAAAAAGCAT) and the primer pair (forward primer, TGGAAGGCTGGGAG CCA; reverse primer, GAAAGCGCAACCGGACG) used for QRT-PCR in analysis of the survivin mRNA were designed using Primer Express 1.0 (Perkin Elmer, Foster City, CA), and synthesized by Applied Biosystems (Foster City, CA). Glyceraldehyde-3-phosphate dehydrogenase (GAPDH) was used as an internal control. A negative control with no template was performed for each reaction series. QRT-PCR reaction was carried out using a LightCycler ${ }^{\mathrm{TM}}$ system (Roche Molecular Biochemicals, Indianapolis, IN). The thermal cycling conditions were $2 \mathrm{~min}$ at $50^{\circ} \mathrm{C}, 30 \mathrm{~min}$ at $60^{\circ} \mathrm{C}, 5 \mathrm{~min}$ at $95^{\circ} \mathrm{C}$, then 40 cycles of $20 \mathrm{sec}$ at $94^{\circ} \mathrm{C}$, and $1 \mathrm{~min}$ at $62^{\circ} \mathrm{C}$. Data were analyzed with LightCycler software.

For quantization of the Ad E1 and E4 genes, total cellular RNA or DNA were extracted from cells or cell cultures in 12/24-well plates using the RNeasy mini RNA extraction kit or a blood DNA kit (Qiagen), respectively. Both RNA and DNA samples were treated with RNase free DNase and DNase free RNase, respectively, to remove possible contamination. The Ad5 E1 gene was detected in RNA samples by using oligo pair forward primer-5'AACCAGTTGCCGTGAGAGTTG and reverse primer-5'CTDGTTAAGCAAGTCCTCGATACA, and probe ORF6-CACAGCTGGCGACGCCCA; and the Ad5 E4 gene was detected in DNA samples by using oligo pair forward primer-5'GGAGTGCGCCGAGACAAC, and reverse primer5'ACTACGTCCGGCGTTCCAT, and probe ORF6-TGGCA TGACACTACGACCAACACGATCT. Negative controls and an internal control were performed for each reaction series as described above.

Transcriptional and transductional evaluations in vitro. Cholangiocarcinoma cells $\left(5 \times 10^{4}\right.$ cells/well $)$ were plated on 24-well plates in $1 \mathrm{ml}$ of medium as described previously. On the following day, cells were infected with recombinant Ads [Ad5-CMV, Ad5-Cox-2, Ad5-CXCR4, Ad5-EGP-2, Ad5HPR, Ad5-SLPI, Ad5-MsLn, and Ad5-S for transcription (Fig. 1) or Ad5.RGD, Ad5.pk7, Ad5.F5/3, and Ad5.pk7.RG for transduction (Fig. 2)] at 100 v.p./cell for $2 \mathrm{~h}$ in $200 \mu \mathrm{l}$ of the medium containing $2 \%$ of FBS. Cells were then washed once with $1 \mathrm{ml}$ of PBS and $1 \mathrm{ml}$ of the medium containing $10 \%$ of FCS was added to each well. After 48-h incubation, cells were washed with PBS, luciferase activity was determined using the Reporter lysis buffer and luciferase assay system of Promega (Madison, WI) following the manufacturer's protocol. Experiments were performed in triplicate and luciferase activity was standardized to the relative light unit (RLU) values of the CMV promoter (the CMV promoter activity is set as $100 \%$ ). The transcriptional levels of the TSP of interest in cholangiocarcinoma cells were evaluated by measuring the expression activity of the luciferase reporter gene driven by different promoter. The transductional levels of the Ads in cholangiocarcinoma cells were evaluated by measuring the 
A

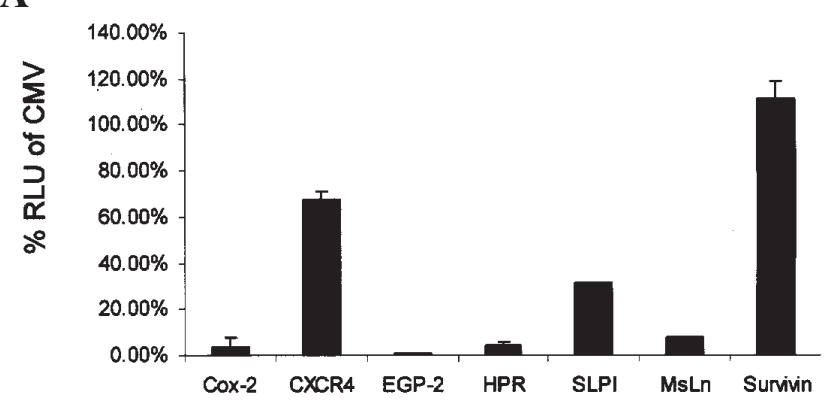

B

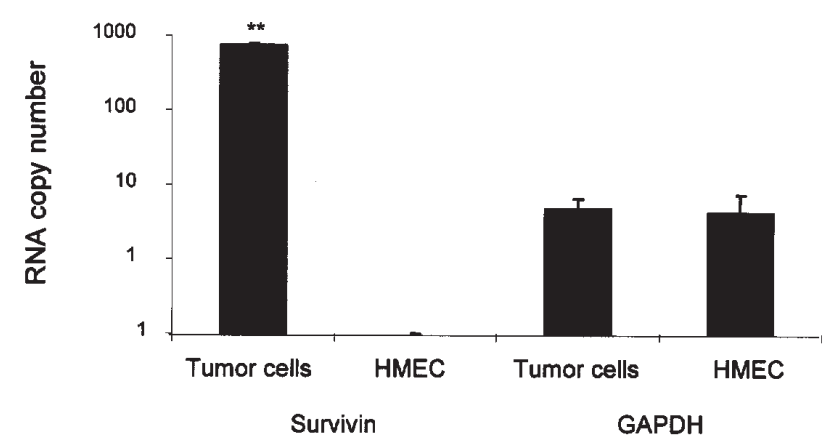

Figure 1. (A) Comparison of promoter activity in cholangiocarcinoma cells. Cholangiocarcinoma cells $\left(5 \times 10^{4}\right)$ were plated on 24 -well plates and infected at an MOI of 100 v.p./cell of Ad5-CMV, Ad5-Cox-2, Ad5-CXCR4, Ad5EGP-2, Ad5-HPR, Ad5-SLPI, Ad5MsLn or Ad5-S, respectively. Luciferase activity was analyzed $48 \mathrm{~h}$ later. Results are shown as relative light units (RLU) of luciferase activity. The \% of luciferase activity $=($ RLU induced by TSP)/(RLU induced by the CMV promoter) $x 100$. The mean value \pm SE of triplicate samples is shown. (B) Survivin and GAPDH gene expression in cholangiocarcinoma and HMEC cells. Both cholangiocarcinoma and HMEC cells $\left(5 \times 10^{4}\right)$ were plated on 24-well plates. After 48-h incubation, the RNAs were isolated from all cells and both the survivin and GAPDH RNA copy number were determined by using RT-PCR. ${ }^{* *} \mathrm{P}<0.01$.

expression activity of the luciferase reporter gene driven with the same CMV promoter, but incorporating a different capsid modification into the Ad fiber region.

Development of CRAd agents. The CRAd genomes were constructed via homologous recombination in Escherichia coli (Fig. 3) as described previously (23). Briefly, DNA fragments containing nucleotides $-230 /+30$ was cut with $B a m H I$ and HindIII restriction endonucleases from the clone pLuc-cyc1.2 which was a gift from Dr F. Li (Buffalo, New York), and subcloned into the plasmid pBSSK (Stratagene, La Jolla, CA) by use of the same restriction sites. A SV40 poly-A (PA) fragment was then cut with $\mathrm{XbaI} / \mathrm{Bam} \mathrm{HI}$ from a pGL3B vector (Invitrogen, Carlsbad, CA) and inserted into the pBSSK/ Survivin by use of the same restriction sites. A generated clone named $\mathrm{pBSSK} / \mathrm{PA} /$ Survivin was used to create shuttle vectors. DNA fragments containing SV40 PA and the survivin promoter (short) were cut with $\mathrm{NotI} / \mathrm{XhoI}$, and subcloned into a pScsE1 plasmid [gift from Dr D. Nettelbeck (Erlangen, Germany)] that contains the E1 gene with the same restriction sites. The shuttle vectors, $\mathrm{pScs} / \mathrm{PA} / \mathrm{S}$, were thus generated.

The Ad vector, pVK503c (26) [a kind gift from Dr V. Krasnykh (Houston, TX)] contains both the E3 gene and a capsid modified RGD. After cleavage with PmeI, the shuttle vectors were recombined with ClaI linerized pVK503c to

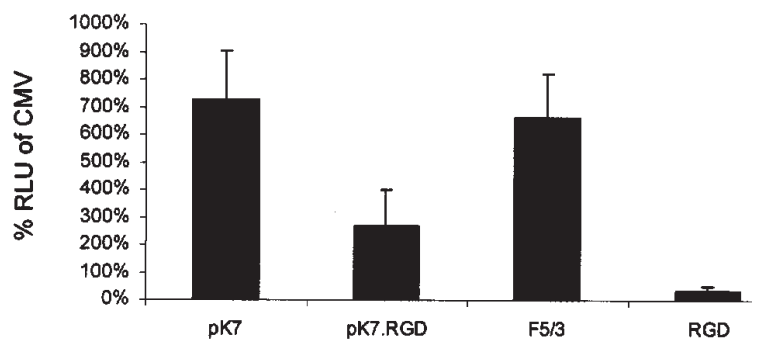

Figure 2. Comparison of transductional activity in cholangiocarcinoma cells with different capsid modified adenovirus vectors. Cholangiocarcinoma cells $\left(5 \times 10^{4}\right)$ were plated on 24-well plates and infected at an MOI of 100 v.p./ cell of Ad5-CMV, Ad5.RGD, Ad5.pk7, Ad5.F5/3 or Ad5.pk7.RGD, respectively. Luciferase activity was analyzed $48 \mathrm{~h}$ later. Results are shown as relative light units (RLU) of luciferase activity. The \% of luciferase activity $=($ RLU induced by TSP $) /($ RLU induced by the CMV promoter $) \mathrm{x}$ $100 \%$. The mean value \pm SE of triplicate samples is shown.

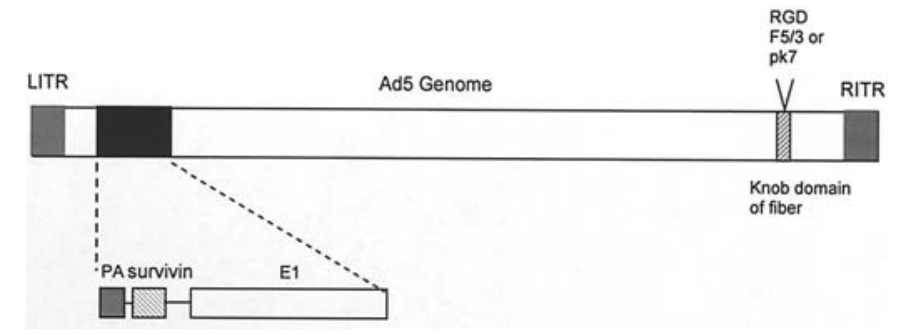

Figure 3. Construction of the CRAd agents. Of the survivin promoter, $260 \mathrm{bp}$ was amplified from the clone pLuc-cyc1.2 and separately constructed in a $\mathrm{pScE} 1$ vector. A poly-A signal sequence (PA) was inserted between the ITR and survivin promoter to terminate the transcription signal from the ITR. Constructed clones were recombinated with pVK503C, AdF5/3 or the Ad5pk7 vector which contain the E3 gene and an RGD4C, F5/3 and pk7 fiber motif to generate the CRAd-S.RGD, CRAd-S.F5/3 and CRAd-S.pk7 constructs, respectively. All three capsid modifications are located in the HI loop region of Ad fiber.

generate a CRAd genome with an RGD-modified fiber. The resultant plasmids encoded the survivin short promoter. CRAds were linearized with PacI and transfected into 911 cells using superfect (Qiagen). Generated viruses were propagated in D65 cells (a glioma cell line in which the survivin gene is overexpressed), and purified by double $\mathrm{CsCl}$ density gradient centrifugation, followed by dialysis against phosphate-buffered saline (PBS) containing 10\% glycerol. The viruses were titrated by plaque assay, and v.p. number was determined spectrophotometrically based on absorbance at a wavelength of $260 \mathrm{~nm}$. The viruses were stored at $-80^{\circ} \mathrm{C}$ until use. CRAd-S.pk7 and CRA-S.F5/3 were generated by a similar method except for the fact that the Ad vectors; Ad.pk7 (14) for CRAd-S.pk7 and pVK500F5/3 (31) for CRAd-S.F5/3, were available in our laboratory. Wild-type Ad5 (Adwt) and Ad5-S (17) were used as replication positive and negative controls, respectively, in the CRAd agent analysis.

Analysis of transduction of CRAd agents in tumor cell lines. Cholangiocarcinoma cells, $10^{5} /$ well, were cultured as discussed above, infected with 100 v.p./cell of Ad5-S, CRAd-S.RGD, CRAd-S.F5/3, CRAD-S.pk7 or Adwt in infection medium containing $2 \% \mathrm{FBS}$, and incubated at $37^{\circ} \mathrm{C}$ in a $5 \% \mathrm{CO}_{2}$ environment. After 3-h incubation, the infection medium was 
removed, the cells were washed three times with PBS to remove uninternalized viruses. DNAs were isolated from cells of each well using the DNeasy tissue kit (Qiagen) and quantitative RT-PCR was performed as described above. Ad E4 gene copy numbers were detected and normalized with human actin gene.

Analysis of replication of CRAd agents in tumor cells. Cholangiocarcinoma cells $10^{5}$ well were cultured as discussed above, infected with 100 v.p./cell of Ad5-S, CRAd-S.RGD, CRAd-S.F5/3, CRAD-S.pk7 or Adwt in infection medium containing $2 \% \mathrm{FBS}$, and incubated at $37^{\circ} \mathrm{C}$ in a $5 \% \mathrm{CO}_{2}$ environment. After 3-h incubation, the infection medium was removed, and the cells were washed three times to remove uninternalized viruses and placed in fresh culture medium with $10 \%$ FBS. Media from triplicate wells were collected 1, 3 , and 9 days later, DNA was extracted from $200 \mu 1$ of media with the DNeasy tissue kit (Qiagen) and quantitative RT-PCR was performed as described above. Ad E4 gene copy numbers were detected and normalized with human actin gene.

In vitro analysis of cytocidal effects. The in vitro cytocidal effects of the CRAd agents were analyzed by determining the viability of the cells with crystal violet staining after infection. Briefly, 2.5×104 cholangiocarcinoma cells/well were plated on a 24-well plate. Cells were infected at 500, 100, 20, 4, 0.8 or 0 v.p./cell with CRAd-S.pk7, CRAd-S.F5/3, CRAd-S.RGD, or Ad5-S in infection medium. Two hours later, the infection medium was replaced with the appropriate complete medium. After 10 days of incubation, the cells were fixed with $10 \%$ buffered formalin for $10 \mathrm{~min}$ and stained with $1 \%$ crystal violet in $70 \%$ ethanol for $20 \mathrm{~min}$, followed by washing 3 times with tap water and air drying. Trypan blue exclusion experiments were also performed as described elsewhere (23).

Replication of CRAd agents on murine xenografts. The tumor cells were verified to have $95 \%$ viability by Trypan Blue exclusion. BALB/c nude mice were subcutaneously inoculated in their flanks with $2.5 \times 10^{6}$ cholangiocarcinoma cells $(n=3 /$ group). When the tumors reached $5 \mathrm{~mm}$ in widest diameter, $5 \times 10^{8}$ v.p. of each viral vector (Adwt, Ad5-S, or CRAd-S.F5/3) were injected intratumorally. The mice were sacrificed at day 1 and day 7 and the tumor samples were harvested. The DNAs were isolated from the tumor samples as described previously. DNA samples from xenografts were stored at $-80^{\circ} \mathrm{C}$ until use. Ad E4 gene copy numbers were detected by QRT-PCR and normalized with human actin, respectively.

Antitumor effect of CRAd agents on mouse xenograft model. $\mathrm{BALB} / \mathrm{c}$ nude mice were inoculated in their flanks with $2.5 \times 10^{6}$ cholangiocarcinoma cells ( $\mathrm{n}=5 /$ group) as described previously. When the tumors reached $5 \mathrm{~mm}$ in widest diameter, $1 \times 10^{9}$ v.p. of each viral vector (Ad5-S, CRAd-S.RGD, CRAdS.F5/3, CRAd-S.pk7 or Adwt) was injected intratumorally. The same dose was repeated after one week. The tumor volumes were monitored twice a week and calculated by the following formula: $1 / 2 x^{2}$, where $x$ is the longest distance and $y$ is the shortest distance of the tumor. The tumor volume was shown as $\mathrm{mm}^{3}$. The statistical analysis was performed by two-way ANOVA.
Analysis of promoter activity in human liver slices. Human liver was obtained from hepatectomy specimens (donor) following liver transplantation. To generate liver tissue slices, tissue was cut in consecutive $0.5-\mathrm{mm}$ thick slices using the Krumdieck tissue slicer. Sequential slices were then cultured in 24-well plates in RPMI medium supplemented with $10 \%$ bovine fetal serum, $100 \mathrm{U} / \mathrm{ml}$ penicillin, $100 \mu \mathrm{g} / \mathrm{ml}$ streptomycin and $5 \mu \mathrm{g} / \mathrm{ml}$ insulin. The tissue slices were infected with 500 v.p./cell of Ad vector, Ad5-CMV or Ad-S with infection medium. After 3-h infection, the liver slices were washed once with PBS and $10 \%$ bovine fetal serum medium was added for 48 -h incubation, at $37^{\circ} \mathrm{C}$, in a humidified atmosphere of $95 \%$ air and $5 \% \mathrm{CO}_{2}$. Two days following infection, luciferase activity was detected by conventional assay and shown as ULR (relative light units) of luciferase activity.

Statistic analysis. Student's test was employed for statistical analysis where $\mathrm{P}<0.05$ was considered to be statistically significant. For tumor growth inhibition experiment, twoway ANOVA was employed.

\section{Results}

Evaluation of tumor-specific promoters in vitro. The activity of all 7 tumor-specific promoters; i.e. the cox-2, CXCR4, EGP-2, HPR, SLPI, MsLn, and survivin promoters, respectively, was evaluated in cholangiocarcinoma cells. The backbone structure of the Ad vectors was identical in all constructs which contained a luciferase reporter gene derived from the pGL3 plasmid (Promega), except for the promoter which specifically drove the luciferase gene expression. The activity of the Ad vectors in tumor cells was normalized to an Ad5-CMV vector which had the same backbone but utilized the CMV promoter. The results are shown in Fig. 1A. Three out of seven TSPs, the survivin, CXCR4 and SLPI promoters, exhibited higher promoter activity than the others in cholangiocarcinoma cells; $111 \%, 67 \%$ and $25 \%$, respectively, in cholangiocarcinoma cells, compared to that of the CMV promoter. The activity of the remaining promoters was $<5 \%$ of the CMV promoter. These data provide evidence that these three promoters have a tumor on phenotype. In addition, survivin gene expression was 7000 RNA copies (S.D. \pm 778 ) in cholangiocarcinoma cells as compared to undetectable (0) levels in the non-transformed HMEC cells as detected by QRT-PCR, which was significant $(\mathrm{P}<0.01)$. Human GAPDH gene expression was detected at a level of 48 (S.D. \pm 7) and 42 (S.D. \pm 29$)$ RNA copies in cholangiocarcinoma and HMEC cells, respectively (Fig. 1B).

Evaluation of capsid modification in vitro. Four capsid modifications, pk7, pk7.RGD, F5/3 and RGD, were generated for viral infectivity enhancement via a CAR-independent pathway as described elsewhere (8). All of the Ad vectors again had similar backbones as described above, the difference being the incorporation of a different modification in the Ad fiber region. The activity of the modified Ad vectors in tumor cells was normalized to an Ad5-CMV vector which had the same backbone as the native Ad5 fiber (Fig. 2). The two Ad vectors with the F5/3 and pk7 modifications exhibited higher reporter activity in the cholangiocarcinoma cells; 664\% (F5/3 
A

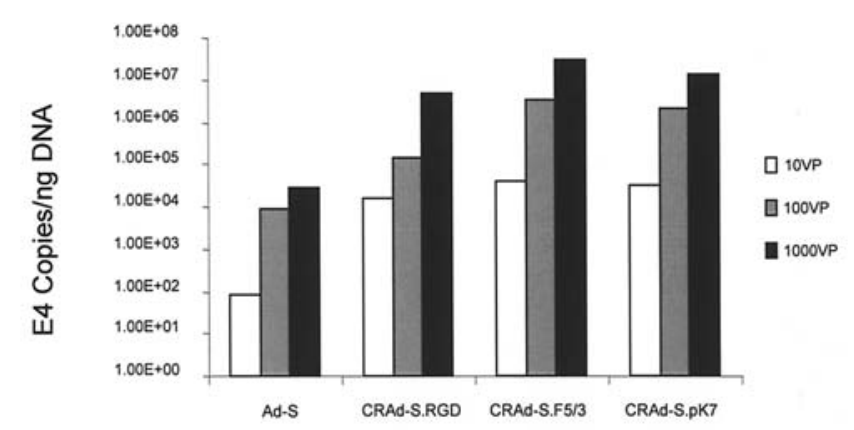

B

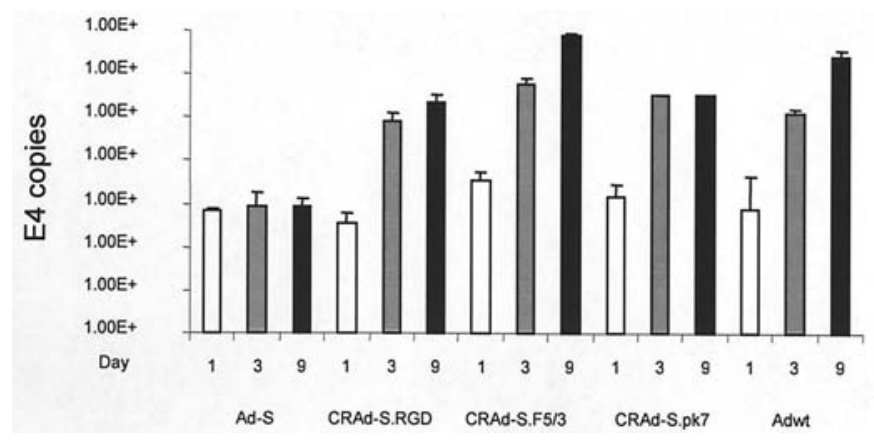

Figure 4. (A) Transductional activity of modified CRAd agents in cholangiocarcinoma cells. The cholangiocarcinoma cells $\left(5 \times 10^{4}\right)$ were plated on 24-well plates and infected at an MOI of 1000, 100 and 10 v.p./ cell of Ad5-S, CRAd-S.RGD, CRAd-S.F5/3 or CRAd-S.pk7, respectively. After 3-h infection, the cells were washed 3 times with PBS to remove uninfected free adenoviral vectors. The DNAs were isolated from these cells and the Ad E4 gene was determined by using RT-PCR. An internal standard, the GAPDH gene, was used for normalizing the DNA amount and the E4 copy number. The ordinate is shown as E4 copies/ng DNA. (B) Replication rates of modified CRAd agents in cholangiocarcinoma cells. The cholangiocarcinoma cells $\left(5 \times 10^{4}\right)$ were plated on 24-well plates and infected at an MOI of 100 v.p./cell of Ad5-S, CRAd-S.RGD, CRAd-S.F5/3, CRAd-S.pk7 or Adwt, respectively. On day 1, 3 and 9 post-infection, $200 \mu 1$ of medium was collected and spun to remove the cell debris. The DNAs were isolated from the media and the Ad E4 gene was determined by using RT-PCR.

modification) and $723 \%$ (pk7 modification), respectively, compared to that of the Ad5-CMV with a native fiber. These data provide evidence that two capsid modifications, pk7 and F5/3, may be good candidates for viral infectivity enhancement in CRAd agents.

Attributes of the survivin CRAds incorporating capsid modifications. Based on these data, we choose the survivin promoter as the tumor-specific promoter to increase tumor specificity and the RGD, F5/3 and pk7 capsid modifications to enhance viral infectivity. The structures of the survivin-CRAd vectors shown in Fig. 3 were constructed by conventional methodologies, propagated in 911 cells, and expanded in D65 cells. The CRAd-survivin vectors have the following characteristics: i) the survivin-CRAds contain the human survivin promoter (nucleotide $-230 /+30$ ) to drive E1 expression (23), the survivin-controlled E1 expression cassette was placed in the original E1 region of the Ad gene; ii) a capsid modification, RGD, F5/3 or pk7, was inserted into the Ad fiber knob region for enhancement of Ad infectivity; iii) the E3 gene was retained in the Ad genome for elevating the oncolysis of the CRAd agents; and iv) a poly-A signal was inserted between the inverted terminal repeat (ITR) and the survivin promoter to stop the non-specific transcriptional activity of the ITR, and to retain the tumor specificity of the survivin promoter. All of the modified structures were verified by PCR and sequencing as described elsewhere (16-18).

The yields of CRAd agents were $5.5 \times 10^{11}, 2.6 \times 10^{12}$ and $1.5 \times 10^{12}$ v.p./ml with a v.p./pfu (plaque-forming units) ratio of 69,41 , and 60 for CRAd-S.RGD, CRAd-S.F5/3 and CRAd-S.pk7, respectively.

Transductional activity of capsid modified CRAds in tumor cells. Most human tumors contain low levels of the coxsackieadenovirus receptor (CAR), the natural endogenous receptor for human adenovirus serotype 2 and 5 (30,32). Capsid modification enhances viral infectivity via a CAR-independent pathway that has been described previously. In this study, we compared three capsid modifications, which were incorporated into the fiber region in the CRAd agents into the cholangiocarcinoma cells. In order to avoid viral replication, we infected tumor cells for $3 \mathrm{~h}$ and immediately isolated DNA from these cells. Between 18 and $24 \mathrm{~h}$ were needed to detect Ad DNA in medium, which corresponds to the life cycle of Ads from entry into tumor cells to release from them. The adenoviral copy number was determined by QRT-PCR and normalized to the house-keeping gene, actin. The data shown in Fig. 4A are the transductional levels (including viral binding and internalization) of three CRAd agents carrying different capsid modifications, i.e. RGD, F5/3 and pk7 in cholangiocarcinoma cells. The results indicate that modified CRAds enhanced the transduction by 100 - to 1000 -fold at both 10 v.p./cell and 1000 v.p./cells compared to non-modified Ad as the control. Also the data showed that the F5/3 and pk7 modifications had somewhat higher transductional levels than that of an RGD modification in tumor cells at all three different doses.

Replication of survivin-CRAds in tumor cell lines. After 18$24 \mathrm{~h}$ of incubation, the CRAd agents replicated in tumor cells and began to be released. Three CRAds plus a negative (Ad5-S) and positive control (Adwt) were used to infect two tumor cell lines at 100 v.p./cell in 24-well plates as described in the Materials and methods. Medium (200 $\mu 1)$ was collected from each well, and spun to remove cells. DNA was isolated from the medium and Ad E4 copy numbers were detected by QRT-PCR, which served as a biomarker for the copy numbers of adenoviruses in the medium. The data shown in Fig. 4B compared E4 copies of Ad vectors in the medium at days 1,3 , and 9 post-infection as described previously. The replication ratios (which were calculated from the E4 copies at day 9/E4 copies at day 1) were 1.3, 635, 2229, 220 and 3745 for Ad5-S, CRAd-S.RGD, CRAd-S.F5/3, CRAd-S.pk7 and Adwt in the cholangiocarcinoma cells, respectively. As we expected, there was no significant replication in negative control, Ad5-S, because of its non-replicative nature. Not surprisingly, the positive (Adwt) control exhibited a higher replication rate compared to those of three CRAd agents.

Survivin-CRAds induce cytotoxicity (oncolysis) in cholangiocarcinoma cells. Conventional oncolysis analysis serves as one of the best modalities for monitoring tumor cell killing. Encouraged by the tumor-specific activity of the survivin 


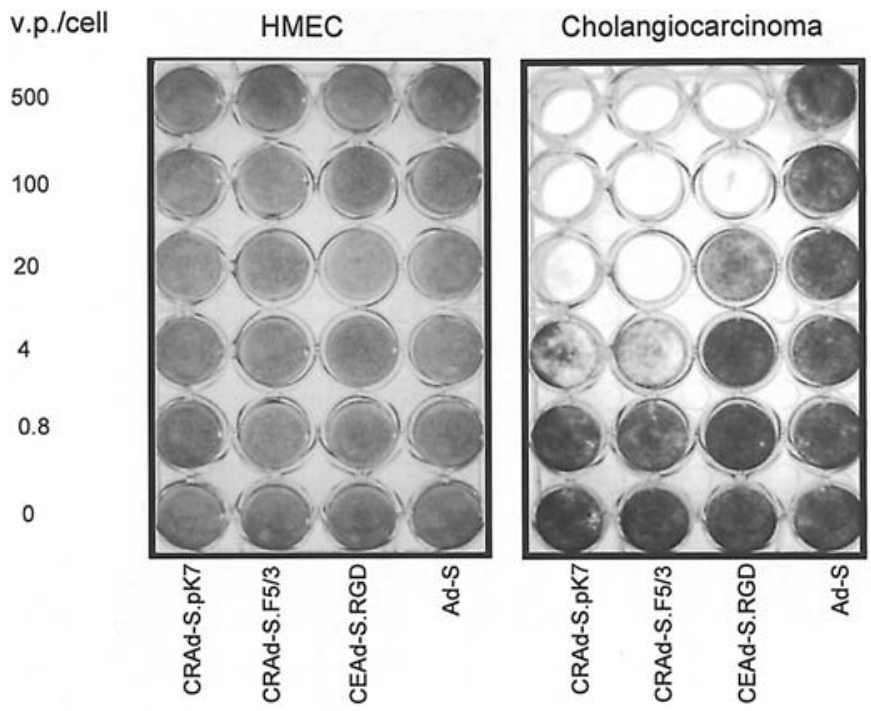

Figure 5. Oncolytic effect of CRAds in cholangiocarcinoma and HMEC cells. Cholangiocarcinoma cells $\left(5 \times 10^{4}\right)$ and HMEC cells, were plated onto 24-well plates, and infected with Ad vector (CRAd-S.RGD, CRAd-S.F5/3, CRAd-S.pk7 or Ad5-S) at the indicated MOIs (500, 100, 20, 4, 0.8 v.p./cell) or mock-infected. After 10-day incubation, cells were stained with crystal violet as described in the Materials and methods

promoter, we used the CRAd-S.RGD, CRAd-S.F5/3 and CRAd-S.pk7 constructs as oncolytic anti-tumor agents in the cholangiocarcinoma cells. Cytotoxicity was evaluated after 10 days of incubation via crystal violet staining (Fig. 5). While the replication-incompetent Ad5-S vector had no cytotoxic effect even at 100 v.p./cell, the survivin-based CRAds induced strong cytotoxicity in cholangiocarcinoma cells. Almost 50\% of cells were killed, even at the smallest dose, 0.8 v.p./cell for CRAd-S.F5/3 and CRAd-S.pk7, and 50\% of cells were killed with CRAd-S.RGD at 20 v.p./cell. As expected, however, no cytoxicity was observed in normal mammary epithelial cells, HMEC, even at the highest dose (500 v.p./cell).

Anti-tumor effect and replication of survivin-CRAds in vivo. The CRAd anti-tumor effect was analyzed in vivo using cholangiocarcinoma cells injected subcutaneously into athymic mice. After establishment and growth of the tumor to $5 \mathrm{~mm}$ in diameter, $1 \times 10^{9}$ v.p. of Ad5-S, CRAd-S.RGD, CRAdS.F5/3, CRAd-S.pk7, Adwt or sham (same volume of PBS) was injected into the tumors, and the injections were repeated once more using the same dose one week later. The tumor growth was monitored over time. The tumor volume data (Fig. 6A and B) shows that there was a significant decrease in tumor volume between control and the treatment groups. At 21 days after treatment, the tumor growth ratio was calculated by dividing the tumor volume at day 21 by the tumor volume at day $1 \times 100 \%$. The tumor growth rates were $438 \%, 313 \%$, $243 \%, 277 \%, 244 \%$ and $540 \%$ for treatment of Ad5-S, CRAd-S.RGD, CRAd-S.F5/3, CRAd-S.pk7, Adwt and sham, respectively. A statistically significant delay $(\mathrm{P}<0.05)$ in tumor growth was achieved for treatment of each of the CRAd agents compared to treatment with PBS or Ad5-S, the non-replicating adenoviral vector. The CRAd agents exhibited a strong antitumor effect similar to or better than Adwt, the replicative control in the cholangiocarcinoma xenograft murine model.
A

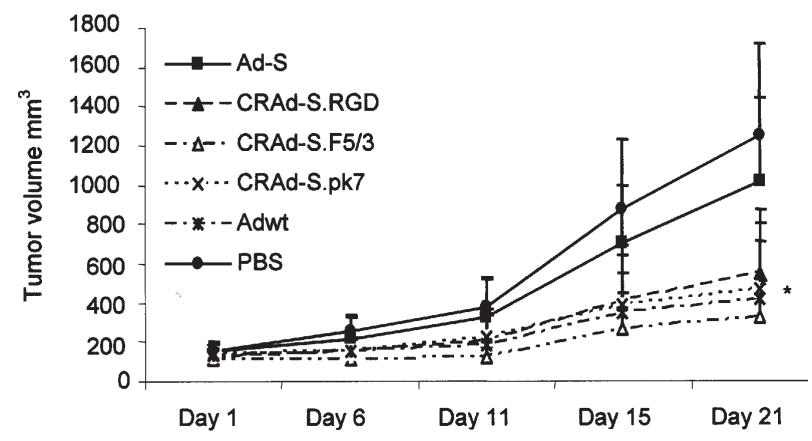

$\mathbf{B}$

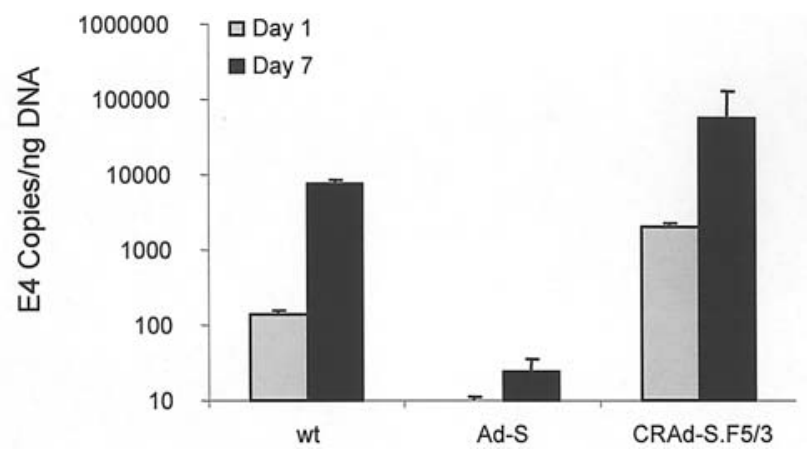

Figure 6. (A) Antitumor effect of CRAd agents on cholangiocarcinoma cells in a mouse xenograft model. Cholangiocarcinoma cells $\left(2.5 \times 10^{6}\right)$ in $200 \mu 1$ of PBS were injected subcutaneously into both flanks of BABL/C nude mice. When the tumor reached $5 \mathrm{~mm}$ in diameter, administration of $1 \times 10^{9}$ v.p. of CRAd agent was performed by intratumoral injection. A second administration was given one week later. The tumor volume was monitored twice a week. The tumor volume was calculated by the formula $1 / 2 \mathrm{xy}^{2}$ where $\mathrm{x}=$ the longest distance of the tumor, and $\mathrm{y}=$ the shortest distance of the tumor. The tumor volumes are shown in the upper panel and the relative tumor growth rates are shown in the lower panel. The relative tumor volume = tumor volume/tumor volume at day one x $100 \%$. ${ }^{*} \mathrm{P}<0.05$. (B) Replication rate of CRAd agents in the cholangiocarcinoma xenograft model. Cholangiocarcinoma cells $\left(2.5 \times 10^{6}\right)$ in $200 \mu 1$ of PBS were injected subcutaneously as described above. When the tumor reached $5 \mathrm{~mm}$ in diameter, administrations of $5 \times 10^{8}$ v.p. of the CRAd agent (Adwt, Ad5-S or CRAd-S.RGD) were performed by intratumoral injection. The mice were sacrificed on day 1 or 7 and then the tumors were removed. DNAs were isolated from tumor tissues and the Ad E4 gene and actin gene levels were determined by using RTPCR. The E4 copy number was normalized as E4 copies/ng DNA.

We also compared the replication rates of CRAd-S.F5/3 to that of the Adwt and Ad5-S vectors. Identical doses of the vectors were injected into the tumor nodules in the murine xenograft model as described in the Materials and methods. The Ad E4 copy number was determined by QRT-PCR as described previously. In this study, the comparison of the E4 copies at day 7 versus day 1 was used to indicate the viral replication rate. The data shown in Fig. $6 \mathrm{~B}$ indicate that the replication rates were 56-, 2.6- and 29-fold over that time period for Adwt, Ad5-S, and CRAd-S.F5/3. Although the capsid modified CRAd, CRAd-S.F5/3, had more than a 10fold infectivity enhancement, comparing the E4 copy number, the replication rate of modified CRAd vector is (29-fold) lower than that of the Adwt (56-fold). The data showed a similar pattern of replication rate in vitro (Fig. 4B).

Survivin promoter activity in human liver slices compared to the CMV promoter. We compared the activity of the survivin 
A.

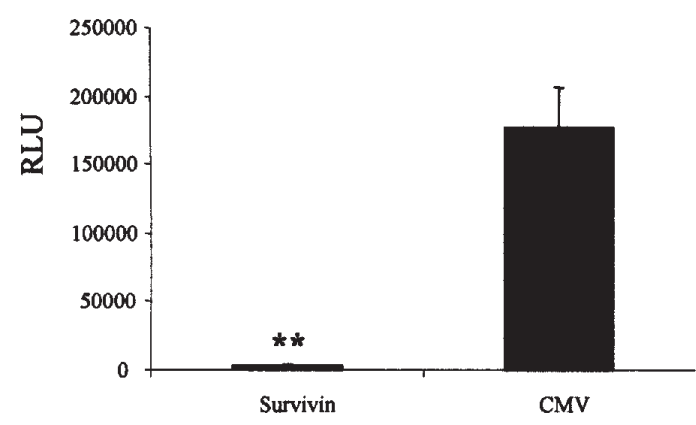

B.

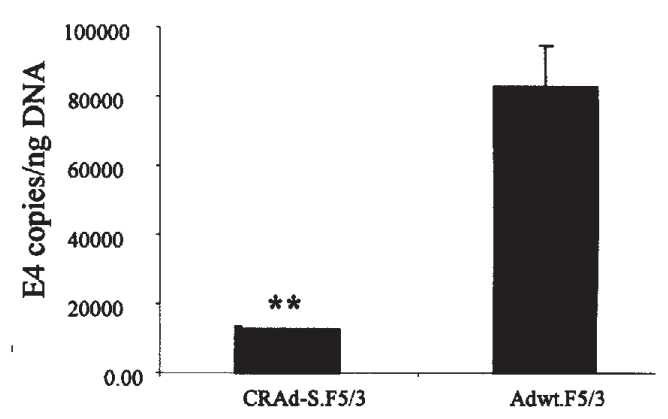

Figure 7. Transcriptional activity of the CMV and survivin promoters in human liver slices. Human liver slices were infected with 500 v.p./cell of Ad vector, Ad5-CMV or Ad5-S (A) and CRAd-S.F5/3 and Adwt.F5/3 (B). Two days following infection, luciferase activity and E4 copy number were detected by using Luciferase assay and real-time PCR as described, respectively. ULR (relative light units) of luciferase expression (A) and E4 copies/ng DNA (B). ** $<0.01$.

promoter and the CMV promoter in human liver slices to evaluate the survivin promoter activity in human liver in relation to the toxicity of the surviving-CRAd agent. The data shown in Fig. 7A demonstrate that the survivin promoter (RLU, 2177 \pm 1892 ) had 81-fold lower activity in liver tissue than that of the CMV promoter (RLU, 177116 \pm 28652 ). These data suggest that the viral replication of survivin-CRAd agents is limited by a liver off promoter in human liver, minimizing the toxicity of the CRAd agent. We also compared the viral replication of CRAd-S.F5/3 to Adwt.F5/3 in human liver slices (Fig. 7B). After two days infection of CRAd-S.F5/3 to Adwt.F5/3, DNA was extracted from the liver slices, and the E4 copies were evaluated by real-time PCR as described in the Materials and methods. The E4 copy numbers of Adwt.F5/3 were 8-fold higher in human liver slices than that of CRAdS.F5/3. Again, the viral replication of CRAd agents was lower level compared to the Adwt.F5/3. The data from Fig. 7A and B indicate that survivin promoter activity results in the lower replication of CRAd agents in human liver. The replication rate of CRAd agents depends on the Ad E1 level which is under-regulated by the tumor-specific promoter, the survivin promoter.

\section{Discussion}

Cholangiocarcinoma is a highly malignant tumor that arises from the bile duct epithelium (33). The tumor usually occurs in older patients and its occurrence in patients younger than 40 years is rare (34). Tumors originating from the large bile duct are discovered early due to their critical location; tumors originating from the small bile ducts do not cause significant biliary obstruction until the tumor itself or metastatic lesions cause obstruction of common bile duct (35). In terms of treatment, complete resection with negative histological margins provides the only hope for long-term survival. Currently, there is no effective adjuvant therapy for this tumor. Cholangiocarcinoma responds poorly to chemotherapy, and radiation therapy and conventional treatments are not adequate for the vast majority of patients with cholangiocarcinoma (36).

Novel therapeutic strategies are warranted for cholangiocarcinoma. Advances in the understanding of growth factor biology, molecular oncology, and tumor immunology have provided the rationale for several strategies for cancer gene therapy (37). Some of these approaches have been applied to the cholangiocarcinoma gene therapy field, including adenovirus-mediated mutant p27kip1 to induce apoptosis of cholangiocarcinoma cells (38), adenovirus-mediated cytosine deaminase suicide gene therapy and 5-fluorocytosine to enhance cytotoxicity in human cholangiocarcinoma (39). Tumor-specific promoter studies (9) and oncolytic herpes simplex virus treatment have also been carried out for this disease $(9,40)$. However, the major challenge has been overcoming inefficient gene delivery at both transductional targeting and transcriptional targeting levels. In this study, we sought to overcome these limitations by use of novel CRAd agents for targeting cholangiocarcinoma.

Replication competent adenoviruses have come into focus as promising novel anti-tumor agents for viral oncolysis and enhanced transfer of therapeutic genes $(41,42)$. Tumor specificity and viral infectivity are the keys to CRAd therapy. The former limits the viral replication in normal host cells, but not in neoplastic cells; the latter enhances the viral infectivity by targeting to tumor cells via a CAR-independent pathway. We, thus, screened 7 tumor-specific promoters and 4 capsid modifications in cholangiocarcinoma cells in this study. Both the survivin and the CXCR4 promoters exhibited higher activity in cholangiocarcinoma cells $(110 \%$ and $70 \%$ activity of the CMV promoter, respecively) (Figs. 1A and 2), however, the survivin promoter had 10-fold lower activity than that of the CXCR4 promoter compared to the CMV promoter in mouse liver when compared to previous data $(16,17)$. Therefore, the survivin promoter has a tumor on/liver off phenotype and was chosen as the tumor-specific promoter to drive Ad E1 expression in CRAd agents against cholangiocarcinoma. Specifically, the liver off status is a critical parameter for clinical application, because wt adenoviruses mainly localize to liver when systemically administered and lead to severe liver dysfunction (43). Adenovirus infection of cells is mediated by attachment of its capsid fiber protein to the cell surface coxsackie adenovirus receptor (CAR) $(44,45)$, followed by interaction of the penton base with $\alpha_{\mathrm{v}}$ integrins that triggers the internalization of viruses (46). However, most tumor cells 
express low levels of CAR, leading to poor infection rates (47). To circumvent this problem, the development of CARindependent Ad vectors which enhance infectivity is critical. We constructed three survivin-CRAds incorporating the capsid modifications RGD, F5/3 or pk7 by which the CRAd agents target to $\alpha_{\mathrm{v}}$ integrins (30), Ad3 receptors CD80/CD86/ CD46 $(48,49)$ or heparan sulfate-containing receptor $(14)$ on the surfaces of tumor cells, respectively, via CARindependent pathways. All of the CRAds which incorporated the capsid modifications had higher transductional activity, especially CRAd-S.F5/3 and CRAd-S.pk7 as compared to the Ad5-S which contained a native fiber with no modification (Fig. 4A). There was a 100- to 1000-fold increase of transductional activity when comparing modified vectors to a non-modified vector at both 10 v.p/cell and 1000 v.p./cell infection doses.

Survivin protein is a novel member of the inhibitor of apoptosis (IAP) protein family, which plays an important role in the survival of cancer cells and progression of malignancies (50). The survivin gene has been described as being selectively expressed in some of the most common human neoplasms, including cancer of the lung (51), pancreas (52), rectum (53), brain (54), and ovary (55). Although the survivin protein is undetectable in normal adult mouse tissue, trace amounts of survivin have been detected in human organs (50). Our data have shown that the survivin promoter has high activity in breast, ovarian cancer and melanoma tumor cells with an extremely low activity in mouse liver (17) and human liver slices (Fig. 7). These data argued that the survivin promoter had a tumor on/liver off phenotype, and thus was a good candidate to be chosen to drive the E1 gene of the CRAd agents in this study, and to restrict the replication of CRAd agents to cholangiocarcinoma tumor cells, not to normal host cells. The survivin promoter is an excellent tumor-specific promoter to target cholangiocarcinoma cells based on the following key points: i) survivin gene expression was detected (7000 RNA copies) in cholangiocarcinoma cells but was undetectable ( 0 copies) in the normal epithelial cell line, HMEC (Fig. 1B); ii) the CRAd agents exhibited strong cell killing in cholangiocarcinoma cells, but not in HMEC cells (Fig. 5); iii) anti-tumor growth experiments indicated that CRAd-S.F5/3 and CRAD-S.pk7 inhibit tumor growth similar to Adwt in in vivo experiments with a $50 \%$ tumor volume as compared to the untreated group (Fig. 6A); iv) there was very low survivin promoter activity in human liver slices as compared to that of the CMV promoter (81-fold) (Fig. 7). All this evidence leads to the incontrovertible conclusion that the survivin promoter has higher activity in cholangiocarcinoma cells but not in normal host cells, HMEC cells or human liver tissue. Thus, the survivin promoter is an excellent TSP to restrict viral replication in cholangiocarcinoma cells and minimize the toxicity to host cells.

Three capsid modifications, RGD, F5/3 and pk7, were used in this study to enhance the viral infectivity of CRAd agents via a CAR-independent pathway. From transductional activity analysis (Fig. 4A), all three capsid modifications were seen to enhance the infectivity in cholangiocarcinoma cells at the three tested doses. The viral infectivity levels were enhanced 100- to 1000-fold at both 10 v.p./cell and 1,000 v.p./ cell compared to the non-modified Ad5-S as control.
Specifically, the F5/3 and pk7 had higher transductional levels than that of the RGD-modified CRAd in cholangiocarcinoma cells. However, the replication ratios were 1.3, 635, 2229, 220, and 3745 for Ad5-Survivin, CRAd-S.RGD, CRAd-S.F5/3, CRAd-S.pk7, and Adwt, respectively, in the cholangiocarcinoma cells (Fig. 4B). Thus, the Adwt had the highest replication rate as compared to the three survivinCRAds. A possible explanation for this is that the capsid modification enhanced the viral infectivity as well as harmed the viral replication under the study conditions, although there is no independent evidence to date to verify this hypothesis.

The use of tumor-specific promoters in cholangiocarcinoma has been previously described by Nagi et al (9). They indicated that the Cox-2 promoter demonstrated a favorable selectivity profile for cholangiocarcinoma while RGD-modification further enhanced transduction efficiency. Many others have also demonstrated that the Cox-2 promoter is a good candidate for G.I. carcinoma gene therapy including that targeted toward human hepatocellular carcinoma (56), gastric cancer (57), colorectal cancer (58), and pancreatic carcinoma (59). Thus, it appears that the Cox-2 promoter acts as a tissue-specific promoter for G.I. cancers as well as G.I. normal tissues including the liver and its bile ducts. More than $90 \%$ of systemically administered adenoviruses localize to and damage the liver. Thus, we compared the promoter activity of both the survivin and Cox-2 promoters in human liver slices and the activity of the survivin promoter was 26-fold lower than that of the Cox-2 promoter (data not shown). However, we could not rule out the possibility that hypoxia up-regulated cyclooxygenase- 2 expression. The authors showed that Cox-2 expression is increased in hypoxia in vitro and in vivo $(60,61)$. Compared to the CMV promoter and viral replication in human liver, both the survivin activity and viral replication of survivin-CRAd were lower in human liver (Fig. 7A and B). Collectively, these findings strongly suggest that this should result in less toxicity of the survivin-CRAd agents to human liver when these agents are applied to clinical trials.

These CRAd agents exhibited a strong tumor cell killing effect in vitro (Fig. 5), replicated in tumor cells (Fig. 6B) and inhibited tumor growth in vivo (Fig. 6A) in this study. Thus, they are promising agents for treatment of cholangiocarcinoma.

In conclusion, we identified the human survivin promoter as a tumor-specific regulatory element for targeting cholangiocarcinoma. CRAd agents armed with the survivin promoter and capsid modifications elevate both tumor specificity and viral infectivity in vitro and in vivo. The survivin-CRAds replicated in tumor cells and inhibited tumor growth in an in vivo murine model. In addition, the survivin promoter had extremely low activity in normal non-transformed epithelium and in human liver. It is apparent that the survivin-CRAds are positioned to be useful for experimental clinical applications for cholangiocarcinoma.

\section{Acknowledgements}

We thank Drs F. Li, D. Nettlbert, V. Kranykh, and H.J. Wu for providing the vectors for this study. Supported by National Institute of Health grants R01 CA83821, R01 CA 94084, 
R01 CA93796, R01 CA93796, K12 HD01261-02, R01 DK063615-01, Dutch Cancer Society grant VU2002-2594 to C.M.F.D, NIH R01 DK063615, NIH P20 CA101955, and a Veterans Administration Merit Award (JMM).

\section{References}

1. Schoenthaler R, Castro JR, Halberg FE and Phillips TL: Definitive postoperative irradiation of bile duct carcinoma with charged particles and/or photons. Int J Radiat Oncol Biol Phys 27: 75-82, 1993.

2. Mittal B, Deutsch M and Iwatsuki S: Primary cancers of extrahepatic biliary passages. Int J Radiat Oncol Biol Phys 11: 849-854, 1985.

3. Pitt HA, Dooley WC, Yeo CJ and Cameron JL: Malignancies of the biliary tree. Curr Probl Surg 32: 1-90, 1995.

4. Pitt HA, Nakeeb A, Abrams RA, et al: Perihilar cholangiocarcinoma. Postoperative radiotherapy does not improve survival. Ann Surg 221: 788-798, 1995.

5. Taniai M, Grambihler A, Higuch Hi, et al: Mcl-1 mediates tumor necrosis factor-related apoptosis-inducing ligand resistance in human cholangiocarcinoma cells. Cancer Res 64: 3517-3524, 2004.

6. Gores GJ: Cholangiocarcinoma: current concepts and insights. Hepatology 37: 961-969, 2003.

7. Alemany R, Gomez-Manzano C, Balague C, et al: Gene therapy for gliomas: molecular targets, adenoviral vectors, and oncolytic adenoviruses. Exp Cell Res 252: 1-12, 1999.

8. Curiel DT: Strategies to adapt adenoviral vectors for targeted delivery. Ann NY Acad Sci 886: 158-171, 1999.

9. Nagi P, Vickers SM, Davydova J, et al: Development of a therapeutic adenoviral vector for cholangiocarcinoma combining tumor-restricted gene expression and infectivity enhancement. J Gastrointest Surg 7: 364-371, 2003

10. Webb HE and Smith CE: Viruses in the treatment of cancer. Lancet 1: 1206-1208, 1970.

11. Nilsson M, Ljungberg J, Richter J, et al: Development of an adenoviral vector system with adenovirus serotype 35 tropism; efficient transient gene transfer into primary malignant hematopoietic cells. J Gene Med 6: 631-641, 2004.

12. Reynolds P, Dmitriev I and Curiel D: Insertion of an RGD motif into the HI loop of adenovirus fiber protein alters the distribution of transgene expression of the systemically administered vector. Gene Ther 6: 1336-1339, 1999.

13. Kawakami Y, Li H, Lam JT, et al: Substitution of the adenovirus serotype 5 knob with a serotype 3 knob enhances multiple steps in virus replication. Cancer Res 63: 1262-1269, 2003.

14. Wu H, Seki T, Dmitriev I, et al: Double modification of adenovirus fiber with RGD and polylysine motifs improves coxsackievirus-adenovirus receptor-independent gene transfer efficiency. Hum Gene Ther 13: 1647-1653, 2002.

15. Glasgow JN, Kremer EJ, Hemminki A, et al: An adenovirus vector with a chimeric fiber derived from canine adenovirus type 2 displays novel tropism. Virology 324: 103-116, 2004.

16. Zhu ZB, Makhija SK, Lu B, et al: Transcriptional targeting of adenoviral vector through the CXCR4 tumor-specific promoter. Gene Ther 11: 645-648, 2004.

17. Zhu ZB, Makhija SK, Lu B, et al: Transcriptional targeting of tumors with a novel tumor-specific survivin promoter. Cancer Gene Ther 11: 256-262, 2004.

18. Lee SE, Jin RJ, Lee SG, et al: Development of a new plasmid vector with PSA-promoter and enhancer expressing tissuespecificity in prostate carcinoma cell lines. Anticancer Res 20: 417-422, 2000 .

19. Huber BE, Richards CA and Krenitsky TA: Retroviral-mediated gene therapy for the treatment of hepatocellular carcinoma: an innovative approach for cancer therapy. Proc Natl Acad Sci USA 88: 8039-8043, 1991.

20. Lu B, Mu Y, Cao C, et al: Survivin as a therapeutic target for radiation sensitization in lung cancer. Cancer Res 64: 2840-2845, 2004.

21. Ikeguchi M, Liu J and Kaibara N: Expression of survivin mRNA and protein in gastric cancer cell line (MKN-45) during cisplatin treatment. Apoptosis 7: 23-29, 2002.

22. Kamizono J, Nagano S, Murofushi Y, et al: Survivin-responsive conditionally replicating adenovirus exhibits cancer-specific and efficient viral replication. Cancer Res 65: 5284-5291, 2005.
23. Zhu ZB, Makhija SK, Lu B, et al: Incorporating the survivin promoter in an infectivity enhanced CRAd-analysis of oncolysis and anti-tumor effects in vitro and in vivo. Int J Oncol 27: 237-246, 2005.

24. Pickens A, Pan G, McDonald JM and Vickers SM: Fas expression prevents cholangiocarcinoma tumor growth. J Gastrointest Surg 3: 374-382, 1999.

25. Pan G, Vickers SM, Phillips JO, et al: Apoptosis and tumorigenesis in human cholangiocarcinoma cells. Involvement of Fas/ APO-1 (CD95) and calmodulin. Am J Pathol 155: 193-203, 1999.

26. Yamamoto M, Davydova J, Wang M, et al: Infectivity enhanced, cyclooxygenase-2 promoter-based conditionally replicative adenovirus for pancreatic cancer. Gastroenterology 125: 1203-1218, 2003.

27. Elkin M, Cohen I, Zcharia E, et al: Regulation of heparanase gene expression by estrogen in breast cancer. Cancer Res 63: 8821-8826, 2003.

28. Barker SD, Coolidge CJ, Kanerva A, et al: The secretory leukoprotease inhibitor (SLPI) promoter for ovarian cancer gene therapy. J Gene Med 5: 300-310, 2003.

29. Breidenbach M, Rein DT, Everts M, et al: Mesothelin-mediated targeting of adenoviral vectors for ovarian cancer gene therapy. Gene Ther 12: 187-193, 2005.

30. Dmitriev I, Krasnykh V, Miller CR, et al: An adenovirus vector with genetically modified fibers demonstrates expanded tropism via utilization of a coxsackievirus and adenovirus receptorindependent cell entry mechanism. J Virol 72: 9706-9713, 1998.

31. Takayama K, Reynolds PN, Short JJ, et al: A mosaic adenovirus possessing serotype Ad5 and serotype Ad3 knobs exhibits expanded tropism. Virology 309: 282-293, 2003.

32. Li Y, Pong RC, Bergelson JM, et al: Loss of adenoviral receptor expression in human bladder cancer cells: a potential impact on the efficacy of gene therapy. Cancer Res 59: 325-330, 1999.

33. Callea F, Sergi C, Fabbretti G, et al: Precancerous lesions of the biliary tree. J Surg Oncol Suppl 3: 131-133, 1993.

34. Okuda K, Kubo Y, Okazaki N, et al: Clinical aspects of intrahepatic bile duct carcinoma including hilar carcinoma: a study of 57 autopsy-proven cases. Cancer 39: 232-246, 1977.

35. Han JK, Choi BI, Kim AY, et al: Cholangiocarcinoma: pictorial essay of CT and cholangiographic findings. Radiographics 22: 173-187, 2002.

36. Chamberlain RS and Blumgart LH: Hilar cholangiocarcinoma: a review and commentary. Ann Surg Oncol 7: 55-66, 2002.

37. Curiel DT, Gerritsen WR and Krul MR: Progress in cancer gene therapy. Cancer Gene Ther 7: 1197-1199, 2000.

38. Yamamoto K, Katayose Y, Suzuki M, et al: Adenovirus expressing p27KIP1 induces apoptosis against cholangiocarcinoma cells by triggering Fas ligand on the cell surface. Hepatogastroenterology 50: 1847-1853, 2003.

39. Stackhouse MA, Pederson LC, Grizzle WE, et al: Fractionated radiation therapy in combination with adenoviral delivery of the cytosine deaminase gene and 5-fluorocytosine enhances cytotoxic and antitumor effects in human colorectal and cholangiocarcinoma models. Gene Ther 7: 1019-1026, 2000.

40. Jarnagin WR, Zager JS, Hezel M, et al: Treatment of cholangiocarcinoma with oncolytic herpes simplex virus combined with external beam radiation therapy. Cancer Gene Ther (in press), 2006.

41. Curiel DT: The development of conditionally replicative adenoviruses for cancer therapy. Clin Cancer Res 6: 3395-3399, 2000.

42. Alemany R, Balague C and Curiel DT: Replicative adenoviruses for cancer therapy. Nat Biotechnol 18: 723-727, 2000.

43. van der Eb MM, Cramer SJ, Vergouwe Y, et al: Severe hepatic dysfunction after adenovirus-mediated transfer of the herpes simplex virus thymidine kinase gene and ganciclovir administration. Gene Ther 5: 451-458, 1998.

44. Philipson L, Lonberg-Holm K and Pettersson U: Virus-receptor interaction in an adenovirus system. J Virol 2: 1064-1075, 1968.

45. Bergelson JM, Cunningham JA, Droguett G, et al: Isolation of a common receptor for Coxsackie B viruses and adenoviruses 2 and 5. Science 275: 1320-1323, 1997.

46. Wickham TJ, Mathias P, Cheresh DA and Nemerow GR: Integrins alpha $\mathrm{v}$ beta 3 and alpha $\mathrm{v}$ beta 5 promote adenovirus internalization but not virus attachment. Cell 73: 309-319, 1993.

47. Miller CR, Buchsbaum DJ, Reynolds PN, et al: Differential susceptibility of primary and established human glioma cells to adenovirus infection: targeting via the epidermal growth factor receptor achieves fiber receptor-independent gene transfer. Cancer Res 58: 5738-5748, 1998. 
48. Short JJ, Pereboev AV, Kawakami Y, et al: Adenovirus serotype 3 utilizes CD80 (B7.1) and CD86 (B7.2) as cellular attachment receptors. Virology 322: 349-359, 2004.

49. Sirena D, Lilienfeld B, Eisenhut M, et al: The human membrane cofactor CD46 is a receptor for species B adenovirus serotype 3 . J Virol 78: 4454-4462, 2004.

50. Bao R, Connolly DC, Murphy M, et al: Activation of cancerspecific gene expression by the survivin promoter. J Natl Cancer Inst 94: 522-528, 2002.

51. Monzo M, Rosell R, Felip E, et al: A novel anti-apoptosis gene: Re-expression of survivin messenger RNA as a prognosis marker in non-small-cell lung cancers. J Clin Oncol 17: 2100-2104, 1999.

52. Sarela AI, Verbeke CS, Ramsdale J, et al: Expression of survivin, a novel inhibitor of apoptosis and cell cycle regulatory protein, in pancreatic adenocarcinoma. Br J Cancer 86: 886-892, 2002.

53. Rodel F, Hoffmann J, Grabenbauer GG, et al: High survivin expression is associated with reduced apoptosis in rectal cancer and may predict disease-free survival after preoperative radiochemotherapy and surgical resection. Strahlenther Onkol 178: 426-435, 2002.

54. Das A, Tan WL, Teo J and Smith DR: Expression of survivin in primary glioblastomas. J Cancer Res Clin Oncol 128: 302-306, 2002.
55. Casado E, Nettelbeck DM, Gomez-Navarro J, et al: Transcriptional targeting for ovarian cancer gene therapy. Gynecol Oncol 82: 229-237, 2001

56. Murata H, Tsuji S, Tsujii M, et al: Promoter hypermethylation silences cyclooxygenase-2 (Cox-2) and regulates growth of human hepatocellular carcinoma cells. Lab Invest 84: 1050-1059, 2004.

57. Chang YJ, Wu MS, Lin JT, et al: Induction of cyclooxygenase-2 overexpression in human gastric epithelial cells by Helicobacter pylori involves TLR2/TLR9 and c-Src-dependent nuclear factorkappaB activation. Mol Pharmacol 66: 1465-1477, 2004.

58. Kim SP, Park JW, Lee SH, et al: Homeodomain protein CDX2 regulates $\mathrm{COX}-2$ expression in colorectal cancer. Biochem Biophys Res Commun 315: 93-99, 2004.

59. Wesseling JG, Yamamoto M, Adachi Y, et al: Midkine and cyclooxygenase-2 promoters are promising for adenoviral vector gene delivery of pancreatic carcinoma. Cancer Gene Ther 8: 990-996, 2001.

60. Saed GM, Munkarah AR, Abu-Soud HM and Diamond MP: Hypoxia upregulates cyclooxygenase- 2 and prostaglandin $\mathrm{E}(2)$ levels in human peritoneal fibroblasts. Fertil Steril 83 Suppl 1: 1216-1219, 2005.

61. Pidgeon GP, Tamosiuniene R, Chen G, et al: Intravascular thrombosis after hypoxia-induced pulmonary hypertension: regulation by cyclooxygenase-2. Circulation 110: 2701-2707, 2004 